\title{
Educación financiera en el proceso de enseñanza-aprendizaje de los estudiantes de Bachillerato del Liceo Francés de San Salvador
}

\author{
Jorge Antonio Portillo López \\ Licenciado en Educación \\ Docente \\ jorge.portillo@catolica.edu.sv \\ ORCID: https://orcid.org/0000-0003-1954-0667
}

Liceo Francés de San Salvador Antoine et Consuelo de Saint-Exupéry

Recepción: 10-11-2020 / Aceptación: 07-01-2021

\section{Resumen}

El estudio analizó los conocimientos de educación financiera, en el proceso enseñanza aprendizaje de los estudiantes de Primer y Segundo año de Bachillerato de Liceo Francés de San Salvador, durante el año 2020. Esto implicó identificar conocimientos de cultura de ahorro, consumo responsable e inversión que poseen los estudiantes. Para ello se realizó una investigación de tipo exploratoria - descriptiva, orientada a tener una aproximación a la educación financiera de los estudiantes de dicha institución.

La investigación se desarrolló con ciento dieciséis estudiantes de ambos sexos, con edades comprendidas entre catorce a dieciocho años. La técnica utilizada fue la encuesta y el instrumento un cuestionario; así también, se entrevistó a docentes de asignaturas afines a la educación financiera para obtener información de la presencia de esta en los programas de estudios.

En los resultados, se encontró que los estudiantes poseen conocimientos sobre el ahorro: guardar dinero por motivos de previsión para cubrir una necesidad o consumo futuro, entre otras. También, existen opiniones divididas en relación a prácticas de consumo responsable de los estudiantes. Además, los estudiantes expresaron conocer lo que significa invertir y sus riesgos, no así sobre los tipos de inversión.

Palabras clave: Educación financiera, ahorro, consumir responsable, inversión.

\begin{abstract}
The study analyzed the knowledge of financial education, in the teaching-learning process of the students of the First and Second year of high school of Liceo Francés of San Salvador, during the year 2020. This implied identifying knowledge of the culture of savings, responsible consumption and investment that students own. For this, an exploratory-descriptive research was carried out, aimed at having an approach to the financial education of the students of this institution.

The research was carried out with one hundred and sixteen students of both sexes, aged between fourteen and eighteen years old. The technique used was the survey and the instrument a questionnaire; Likewise, teachers of subjects related to financial education were interviewed to obtain information on its presence in the study programs.

In the results, it was found that students have knowledge about saving: saving money for forecasting reasons to cover a need or future consumption, among others. Also, there are divided opinions regarding responsible consumption practices by students. In addition, the students expressed knowing what it means to invest and its risks, not about the types of investment.
\end{abstract}

Key words: Financial Education, saving, consume responsibly, investment. 


\section{Introducción}

Frecuentemente, los seres humanos toman decisiones sobre transacciones financieras, estando conscientes o no sobre el impacto a futuro que estas pueden tener sobre las finanzas personales, familiares, empresariales, entre otros. Estas decisiones y acciones le acompañan a lo largo de la existencia, volviéndose parte de la cotidianeidad e importantes para la vida.

Decidir comprar un producto de consumo, solicitar un crédito, adquirir una tarjeta de crédito, membresías de ocio, apertura de una cuenta de ahorro, adquirir una vivienda, comprar seguros (de vida, salud, casa, automóvil, entre otros.); cuotas voluntarias para incrementar fondos de pensión, compra de acciones, pagos de impuestos entre otros; son algunos ejemplos de decisiones financieras. Todas y cada una de ellas generan pasivos o activos financieros, dependiendo del uso que se le dé; la adquisición de bienes pasivos requiere de otros gastos y la adquisición de bienes activos generan ingresos.

Existe cierta conciencia sobre el bajo nivel de formación financiera en el país, e incluso se han tomado algunas medidas, pero ¿están los jóvenes adquiriendo los conocimientos y competencias financieras que necesitan para su vida como adultos? ¿Los programas educativos de El Salvador incluyen la educación financiera como un eje transversal? ¿Cómo se incorpora la Educación financiera como eje transversal en el proceso de enseñanza apren- dizaje de los estudiantes de Primer y Segundo año de Bachillerato de Liceo Francés de San Salvador en el año 2020?

La educación financiera se entiende como el (...) proceso por el cual las personas, a lo largo de su ciclo de vida, mejoran su comprensión del mundo financiero, conceptos y riesgos y, a través de la información, la enseñanza y el asesoramiento objetivo, desarrollan las habilidades, actitudes y confianza para adquirir mayor conciencia de los riesgos y oportunidades financieras, tomar decisiones informadas, saber dónde acudir para pedir ayuda o procurar la protección de sus derechos, y tomar cualquier acción eficaz para mejorar su bienestar y el de la sociedad. (Comisión asesora para la educación financiera, 2016).

En este sentido, el proceso formativo no debe ser marginal en la educación formal: se requiere expansión y profundización de la enseñanza de cuestiones económicas y financieras, que son de uso cotidiano. Es necesario preparar a los estudiantes para enfrentar el complejo mundo económico, marcado por el crecimiento de productos y servicios financieros; asimismo, formarlos para que sean consumidores responsables: críticos, informados, reflexivos, hace valer sus derechos, empáticos y solidarios con los demás y el medioambiente. La competencia financiera es una habilidad esencial en la formación de ciudadanos críticos y responsables. Por este motivo, desde 2012, la prueba internacional 
PISA $^{1}$ evalúa el conocimiento de estudiantes de quince años en temas como el dinero y las transacciones; la planificación y gestión de las finanzas, y el riesgo y el beneficio, entre otras.

Los programas educativos salvadoreños: Plan Nacional de Educación 2021 (2005) y Plan El Salvador Educado (2016) contemplan y enfatizan como pilar la educación integral de niños y adolescentes. El enfoque del currículo salvadoreño se declara constructivista, humanista y socialmente comprometido; y trabaja en función del desarrollo de competencias que propicien en el alumnado la adquisición de los aprendizajes significativos (saberes), por medio de la solución de problemas contextualizados y el desarrollo del pensamiento crítico (Plan 2021, 2005, p. 20). Asimismo, se opta por una educación de calidad, orientada a reducir los niveles de desigualdad y exclusión, que rompe con los mecanismos que perpetúan estas condiciones en los grupos y las familias de menores ingresos, marginadas de los derechos sociales, para lograr una más rápida integración del país en la sociedad y la economía del conocimiento e incrementar la competitividad (Plan El Salvador Educado, 2016, p.9).

No obstante, estas declaraciones en los planes educativos no se consideran, de forma explícita, educación financiera; ni en los contenidos de los programas de Matemática y Estudios Sociales y Cívica; ni como asignatura especifica de Educación Financiera. Esta temática existe en el programa del nivel de Bachillerato el Curso de Habilitación Laboral como materia complementaria u optativa; cuyo propósito es permitir al egresado de Bachillerato su incorporación al mundo laboral, ya sea como empleado o como emprendedor.

En El Salvador, en el año 2008, fue implementada la estrategia nacional para la educación financiera (Programa de Educación Financiera de El Salvador); diseñada como una iniciativa de colaboración interinstitucional entre el Banco Central de Reserva, Superintendencia del Sistema Financiero, Superintendencia de Valores y Superintendencia de Pensiones, y el Instituto de Garantía de Depósitos. (OEDC -CAF, 2013, p.45). El establecimiento de la estrategia se consideró fundamental, dado el nuevo rango y la creciente sofisticación de los productos financieros al alcance de los ciudadanos del país, producto de la globalización y la innovación financiera. Un motivo adicional fue la necesidad de racionalizar la existencia de programas bajo un único marco, de manera que se aumentara la eficiencia de los recursos utilizados y se coordinara su cobertura. En cuanto a la iniciativa, su parte institucional reconocía - desde el principio la necesidad de involucrar al sector privado y alinear sus esfuerzos con aquellos realizados por las autoridades públicas y el Gobierno, en cuanto a la educación financiera. (OEDC -CAF, 2013, p.45).

El documento oficial esboza que la misión de la estrategia de educación financiera es la

1. PISA es el acrónimo en inglés de Programa para la Evaluación Internacional de Alumnos, impulsada por la OECD. 
promoción del conocimiento de productos y servicios; así como sus beneficios, riesgos y costos, y el fortalecimiento de la comprensión de los servicios financieros que conduzcan a una mejor toma de decisiones financieras. $\mathrm{Y}$, en última instancia, a una mejor calidad de vida de los consumidores (OEDC -CAF, 2013, p.46).

La vulnerabilidad socioeconómica de la sociedad contemporánea frente a los diversos vaivenes de la economía internacional, principalmente a partir de las últimas crisis económicas sucedidas en distintas partes del mundo, hace que la educación financiera sea un contenido o eje transversal en los currículos nacionales. Diversos factores apremian:

- Alto nivel de endeudamiento de las personas

- Dependencia de los productos financieros

- Alto grado de desconocimiento y de información sobre aspectos económicos

- Alto nivel de consumismo

- Nuevas formas de entender y manejar el dinero.

Todo esto hace necesario considerar la educación financiera como un elemento fundamental para reflexionar, ya que es en este espacio donde los estudiantes desarrollan conocimientos, competencias y actitudes iniciales que determinan su desarrollo económico posterior. Así pues, El Salvador debe asumir el desafío de incorporar contenidos de educación financiera en el currículum escolar, de modo que los niños y jóvenes conozcan y puedan manejar los diferentes servicios financieros para una satisfactoria incorporación al mundo económico y financiero.

A partir de estas consideraciones se decidió realizar la investigación sobre los conocimientos de educación financiera, como eje transversal en el proceso enseñanza aprendizaje de los estudiantes de Primer y Segundo año de Bachillerato de Liceo Francés de San Salvador durante el año 2020. De tal manera que se obtuvo una aproximación a esta realidad tan importante para los niños y jóvenes como consumidores de servicios y protagonistas de transacciones financieras.

\section{Metodología}

La investigación fue de tipo exploratorio - descriptivo, orientada a tener una aproximación o conocimiento de la educación financiera de los estudiantes de Primer y Segundo año de Bachillerato, del Liceo Francés de San Salvador Antoine et Consuelo de Saint-Exuperio durante el año 2020. El estudio se desarrolló en seis secciones: tres de Primer año y tres de Segundo año de Bachillerato. La cantidad de estudiantes que participaron en este estudio fue de 116, de una matrícula total de 147 estudiantes del año lectivo en cuestión. Participaron tanto estudiantes del sexo femenino como del masculino, cuyas edades oscilaron entre catorce a dieciocho años.

Se implementó un cuestionario elaborado en Google forms como instrumento, a través de la técnica de la encuesta para recabar infor- 
mación entre los estudiantes. La estructura del cuestionario fue de veinte preguntas, de las cuales diecinueve fueron cerradas $y$ una abierta.

En relación a los docentes, la técnica utilizada fue la entrevista; y estuvo dirigida a quienes impartían las asignaturas de Sciences de la Vie et la terre, Economía y Estudios Sociales. Esta técnica permitió obtener información sobre temas o contenidos presentes, explícita o implícitamente, en los programas de estudios de las respectivas asignaturas. También se utilizó una guía para orientar la entrevista con los docentes.

Respecto al análisis e interpretación de la información, la misma se sometió a un estudio sistemático, teniendo en cuenta los objetivos del mismo. En este sentido, se extrajo el significado, interpretación y conclusiones de la información brindada por los estudiantes y docentes del Liceo Francés de San Salvador como material de discusión y análisis.

\section{Resultados}

De acuerdo a los resultados, los estudiantes manifestaron tener conocimiento sobre el ahorro: guardar dinero para el futuro (55.17\%); ahorrar dinero para emergencias (17.24\%); no gastar dinero innecesariamente (17.24\%) y guardar dinero para inversiones (8.62\%). El ahorro como una capacidad de compra diferida, es decir, no comprometida para ser gastada con carácter inmediato; guardar una cantidad de dinero por motivos de previsión con la esperanza de cubrir una necesidad o consumo futuro, ya sea por razo- nes de espera hasta alcanzar el capital que necesita para una acción concreta, o para invertir. En relación a la presencia o inclusión en los programas de estudio, la cultura del ahorro es un contenido presente, implícitamente, en el programa de Estudios Sociales de Primaria como parte de la temática "Presupuesto familiar: Plan y distribución”. Por su parte, los docentes de Educación Básica y Media de Estudios Sociales, que se entrevistaron, refieren que la temática de cultura del ahorro no está presente en los programas de su materia. En la asignatura de Sciences de la Vie et la Terre (SVT), el ahorro está más enfocado a la optimización de los recursos naturales para evitar el desperdicio y no tanto como ahorro económico.

Al analizar la asignatura de Economía del programa francés, el docente mencionó que existe la temática: "Le taux d'intérêt, le revenu disponible des ménages se répartit entre consommation et épargne et qu'ils peuvent dégager des besoins ou des capacités de financement" (La tasa de interés, la renta disponible de los hogares se divide entre consumo $\mathrm{y}$ ahorro y pueden identificar necesidades o capacidades de financiación). En este sentido, se puede inferir que los estudiantes han recibido conocimientos sobre cultura del ahorro. No obstante, estas declaraciones en los programas de educación nacionales, no se consideran de forma explícita; ni en los contenidos de los programas de Estudios Sociales y Cívica, ni como asignatura especifica de Educación Financiera. 
El $86.2 \%$ de los estudiantes entrevistados manifestaron que sus padres sí les inculcan la práctica del ahorro dentro del núcleo familiar, mientras que para un $13.8 \%$ es todo lo contrario. No obstante, aunque un amplio porcentaje de los estudiantes aseguró que en su hogar se les inculca sobre el ahorro, ante la pregunta de si poseen este hábito en concreto, solo el $79.1 \%$ de ellos considera que lo practican.

Por su parte, los docentes manifestaron que los estudiantes poseen muy poco o nada de conocimientos sobre cultura de ahorro. En palabras de uno de los entrevistados, esto “(...) es más que evidente que carecen de ello (hábito del ahorro) y se puede apreciar en la poca importancia que le dan al cuido de sus pertenencias y el desperdicio de recursos".

En esencia, los programas de Educación Financiera ponen énfasis en el ahorro como un elemento esencial en la actuación financiera, de ahí que el ahorro sea considerado por los docentes como un hábito a inculcar entre los niños y jóvenes. Sin embargo, es importante potenciar y profundizar los esfuerzos actuales para la divulgación de programas de ahorro en una temprana edad, como forma de inculcar el hábito del ahorro.

En relación al proceso presupuestario en la familia, llama la atención que un $54.4 \%$ de los estudiantes expresaron que dentro de sus hogares no se elabora un presupuesto; contrario a un
45.6\% de ellos sí lo elaboran. Elaborar un presupuesto es una técnica que colabora en la gestión analítica de una determinada empresa o familia, y puede llegar a constituir una nueva filosofía de administración gerencial sistemática.

El proceso presupuestario se apoya sobre las previsiones elaboradas en función de las condiciones que configuran el entorno empresarial o familiar. Con base en esto, los directivos de la entidad - para el caso en estudio los miembros de la familia - realizan periódicamente una comparación entre los datos presupuestados y los reales, con el fin de poder detectar y analizar las desviaciones producidas.

En cuanto a recibir una mesada ${ }^{2}$, un amplio porcentaje de los estudiantes respondieron no recibirla (75.9\%); pero un $24.1 \%$ aseguró que sí percibe este dinero. Dicha práctica como puede ser un instrumento importante en la educación financiera - más que por la cantidad en sí misma - por la acción de recibirla, ya que le brinda al niño y/o adolescente la oportunidad de ir aprendiendo a administrar el dinero. Esta práctica favorece el desarrollo de autocontrol, tolerancia a la frustración, toma de decisiones y planificación. La mesada es una estrategia $\mathrm{u}$ oportunidad para que cada estudiante se ejercite en el establecimiento de una planificación financiera, que le permita alcanzar las competencias que le faciliten el acceso y uso consciente e informado de los servicios financieros. En resumen, ser un consumidor responsable.

2. Entiéndase como el dinero que los padres dan al niño o joven, de manera periódica o regular, para sufragar gastos o gustos personales. Esta retribución económica no está condicionada a su conducta, ni al rendimiento académico o la colaboración dentro de las tareas del hogar. 
Otro aspecto a considerar es la compra responsable, la cual considera los aspectos de la necesidad que se requiere satisfacer, el presupuesto, así como los requisitos ambientales y sociales relacionados con el ciclo de vida del producto/servicio (este tipo de compra también es conocida como una compra sustentable). Para Bianchi, Ferreyra y Kosiak, (2014), esto es un comportamiento reflexivo y racional del consumidor responsable: planificar las compras; informarse de precios y ofertas; valorar la necesidad de los productos. De acuerdo a los estudiantes, dentro de sus familias, solo el 73.9\% de ellos comparan precios al momento de hacer compras; mientras que un $26.1 \%$ no lo hace.

En cuanto a la pregunta sobre el conocimiento de la importancia de la inversión - entendida en término generales como la utilización de los recursos en el sector productivo o de capitales, con el objetivo de lograr beneficios o ganancias - los estudiantes manifestaron diversas posturas (ver tabla 1).

La práctica de la inversión es significativa porque a raíz de ella se puede obtener libertad financiera. Para realizar una inversión se debe contar con recursos financieros (dinero). Las inversiones aumentan por medio de los intereses, dividendos, acciones y apreciación de los bienes (incremento del valor).

\section{Discusión}

Cada persona atraviesa a lo largo de su vida distintas etapas en las que sus necesidades económicas van variando. Un plan integral de educación financiera considera las diversas situaciones en las que una persona puede necesitar información y formación, antes de tomar una decisión en este ámbito; como, por ejemplo, la financiación de los estudios, la solicitud de una hipoteca, la elaboración de un plan de ahorro, entre otros.

Tabla 1. Rango de edad de los estudiantes encuestados

\begin{tabular}{|l|l|c|}
\hline \multirow{4}{*}{ Invertir } & \multicolumn{1}{|c|}{ Criterio expresado } & Porcentaje \\
\cline { 2 - 3 } & Es un colchón económico frente a emergencias & $34.5 \%$ \\
\cline { 2 - 3 } & Es una forma fácil de hacer más dinero & $30.2 \%$ \\
\cline { 2 - 3 } & Evita el gasto innecesario del dinero & $17.2 \%$ \\
\cline { 2 - 3 } & \begin{tabular}{l} 
Por la tranquilidad de utilización en un momento necesario \\
\cline { 2 - 3 }
\end{tabular} & $\begin{array}{l}\text { Es un colchón económico frente a emergencias y una mejor } \\
\text { solución a largo plazo. }\end{array}$ \\
\hline \multicolumn{2}{|c|}{ Total } & $0.9 \%$ \\
\hline
\end{tabular}


En el contexto de una formación integral, la educación financiera es importante para todas las personas, y no solo para quienes tienen una alta participación en los mercados financieros o se dedican a tareas de inversión. Por esto, la importancia de la competencia financiera se reconoce cada vez más en el ámbito escolar, en cuanto que un número gradual de países están elaborando nuevos currículos y aplicando estrategias de aprendizaje centradas en este campo (Pisa, 2012). En este sentido, los centros educativos están en una posición ventajosa para lograr que la educación financiera llegue y sea accesible a todos los grupos demográficos, sin discriminación alguna; y alcance a niños y jóvenes procedentes de todos los sectores o grupos sociales (incluidos los más vulnerables); tratando de romper el ciclo de desconocimiento financiero generacional y promoviendo así la igualdad de oportunidades en materia de formación financiera (Pisa, 2012, p.9).

La educación financiera contribuye a una mejor calidad de vida en la construcción continua del conocimiento; en el respeto de los valores, el desarrollo de destrezas, habilidades, actitudes y en la toma de decisiones dentro de temas que tienen que ver con la organización de un presupuesto; la distribución de gastos, las inversiones en educación, la adquisición de bienes y servicios, la adquisición de préstamos; así como la previsión de un ahorro para una jubilación digna y otros temas financieros.

Un elemento importante en educación financiera es el ahorro y sus implicaciones, tanto a nivel informal como formal: los aspectos conceptuales y la importancia del ahorro; los intereses; cómo calcular y manejar los intereses; las cuentas de ahorro y sus tipos; cómo aumentar los ahorros; la inversión; el cálculo a futuro de los ahorros actuales y cómo identificar metas para ahorrar. De esta forma, las decisiones económicas futuras que tendrán que afrontar los jóvenes suponen un reto mayor que en el pasado reciente, teniendo en cuenta la complejidad de los productos y servicios de los mercados financieros actuales. Por ello es esencial que los alumnos empiecen a desarrollar habilidades financieras desde edades tempranas, a fin de prepararles para vivir, el día de mañana, de manera independiente; comprendiendo el complejo contexto económico que les rodea y participando activamente en él, sabiendo desenvolverse y tomando decisiones inteligentes (Pisa, 2012, p.8).

La educación financiera, además, consiste en transmitir conocimiento y desarrollar habilidades, orientadas a mejorar la toma decisiones de los individuos y de sus hogares en materia financiera. En última instancia, es producir un cambio de comportamiento de los agentes económicos, de manera que mejoren la forma en la que administran sus recursos financieros (Gómez-Soto, 2009, p.8). De acuerdo con esto, la educación financiera incluye el consumo responsable; es decir, la generación de estrategias que tienen como meta satisfacer las necesidades del ser humano de una manera especial, cuestionando al mismo tiempo lo que ese consumo puede estar dañando a los 
recursos naturales, y los problemas de contaminación que se están generando y se pueden generar (Gutiérrez, 2018).

Se debe tener conocimiento de cómo se debe consumir con responsabilidad y tener en cuenta las decisiones que se toman cada vez que se hace una compra. Estas últimas deben hacerse de forma consciente para que cada vez exista menos contaminación en el mundo; se perciban cambios de hábitos en la vida cotidiana $y$, a nivel personal, no ser víctima del consumo exacerbado que se impone en la sociedad actual. El consumidor responsable está muy bien informado, analiza las etiquetas de los productos que adquiere y reconoce el producto ecológico (Flores, 2019).

El consumo responsable invita a disociar el deseo de la necesidad, a fin de lograr - en un contexto real - la toma de decisiones correctas en la gestión de la economía personal y familiar; y la responsabilidad social del cuido del medioambiente. Los consumidores con un conocimiento ajustado de sus necesidades y de los productos disponibles están en facultad de tomar mejores decisiones, adquiriendo los servicios y productos más adecuados. Así, todo esfuerzo empleado en un plan de Educación Financiera debe emplearse en formar personas críticas con capacidad de análisis y de autonomía; que en el futuro puedan tomar las decisiones que más les convienen, sin verse influenciadas por la publicidad nociva o intereses comerciales de las entidades bancarias, que solo buscan su propio beneficio.

\section{Visión a futuro}

Por otra parte, hay que considerar que la inversión - como instrumento para aumentar el ahorro - constituye otro de los aspectos en la educación financiera, especialmente sus elementos conceptuales, enfoques, tipos de inversión y los criterios para realizarla; así como sus instrumentos. También, es importante considerar aspectos relacionados con las instituciones o sociedades de inversión, los portafolios y los primeros pasos a seguir por parte de los principiantes para realizar una inversión; entendiendo al mismo tiempo por qué este tipo de actividades fracasan y los riesgos de la misma.

Una inversión es cualquier instrumento en el que los inversionistas depositan fondos con la expectativa de que genere ingresos positivos y/o conserve o aumente su valor. Los rendimientos de la inversión se reciben como ingresos corrientes o aumento del valor. La educación financiera, en este sentido, debe permitir formar a los niños y jóvenes a hacer uso de esta herramienta; de tal modo que les permita conducirse por medio de planes bien desarrollados, establecidos para lograr metas específicas de sus finanzas personales a futuro.

Si bien es cierto existen unos primeros pasos en la implementación de la educación financiera, es importante que el Ministerio de Educación de El Salvador - desde un enfoque de competencias - promueva la competencia financiera y su inclusión en el currículo; así como su implementación en el aula de ma- 
nera explícita, como asignatura o como contenido dentro del programa de asignaturas ya existentes. Es importante que esta entidad profundice en la temática de Educación Financiera, ya que los niños y jóvenes, muy probablemente, tendrán que asumir riesgos financieros más frecuentes, tanto por el aumento de la esperanza de vida como por la disminución de los beneficios del trabajo, $y$ una incertidumbre mayor en las posibilidades de empleo (Pisa, 2012).

Así también, es necesaria la formación de los docentes en el área de Educación Financiera, ya que es un pilar fundamental para conseguir el éxito en la introducción de la educación financiera en el sistema educativo; es decir, un plan integral de educación financiera que considere las diversas situaciones en las que una persona puede necesitar información y formación al tomar este tipo de decisiones.

Por otro lado, se sugiere al Liceo Francés de San Salvador la implementación de un programa de educación financiera que se ajuste a su currículo y a su dinámica académica. Es esencial que los alumnos empiecen a desarrollar competencias y habilidades financieras desde edades tempranas. Se trata de prepararles para vivir el día de mañana de manera independiente, que comprendan el complejo contexto económico que les rodea y que participen activamente en él, sabiendo desenvolverse y tomando decisiones inteligentes (Pisa, 2012).
Existe una relación entre la competencia financiera y el contexto familiar económico y educativo: las personas que son más competentes desde el punto de vista financiero, proceden en gran parte de entornos con un alto nivel educativo y de familias que poseen una amplia variedad de productos financieros (Lusardi, 2010, citado en Pisa, 2012). En este sentido, se recomienda al Liceo Francés de San Salvador, la implementación de un programa de educación financiera en la Escuela de Padres y Madres, de modo que estos sean orientados en este aspecto, a fin de que posean mejores herramientas para educar financieramente a sus hijos.

Sin duda, esta investigación da pie a nuevos estudios de carácter más extensivo, que ahonden en la importancia y necesidad ingente de la educación financiera, especialmente en el acceso y manejo de los recursos financieros, independientemente o al margen de las instituciones bancarias; sino más direccionados en la línea de defensoría al consumidor, a fin de formar ciudadanos críticos, conscientes y responsables en el manejo de sus recursos financieros. 


\section{Referencias}

Bianchi, C.; Ferreyra, S. y Kosiak, G. (2014). Consumo responsable: diagnóstico y análisis comparativo en la Argentina y Uruguay. Escritos Contables y de Administración, 4 (1), 43 -79. https://revistas.uns.edu.ar/eca/article/view/280

Comisión Asesora para la Inclusión Financiera (2016). Estrategia Nacional de Educación Financiera, Inclusión Financiera, Chile, https://bibliotecadigital.mineduc.cl/bitstream/handle/20.500.12365/2167/mono-991.pdf

Flores-Domínguez, A. (2019). El consumo responsable y el estatus. Vincula Tégica EFAN, 5 (2), 107-114, http://www.web.facpya.uanl.mx/Vinculategica/vinculategica_5/10\%20FLORES.pdf

Gómez-Soto, F. (2009). Educación financiera: retos y lecciones. A Partir de Experiencias Representativas en el Mundo, Proyecto Capital, Bogotá, http://www.asbasupervision.com/en/ $\mathrm{bibl/financial-inclusion/financial-literacy/177-educacion-financiera-retos-y-lecciones-a-}$ partir-de-experiencias-representativas-en-el-mundo/file

Ministerio de Educación, Cultura y Deporte (2014). PISA 2012 Competencia Financiera. Informe español, https://sede.educacion.gob.es/publiventa/pisa-2012-competencia-financierainforme-espanol/ensenanza-finanzas/20001

Ministerio de Educación de El Salvador (2005). Plan Nacional de Educación 2021, https://siteal. iiep.unesco.org/bdnp/3382/plan-nacional-educacion-2021

Ministerio de Educación de El Salvador (2016). Plan EL Salvador Educado, https://www.unicef. org/elsalvador/media/1236/file

OCDE (2015). Résultats du PISA 2012: Les élèves et l'argent (Volume VI): Les compétences en culture financière au XXIe siècle, PISA, OCDE Publishing Paris, http://dx.doi. org/10.1787/9789264243385-fr

OEDC - CAF (2013). La educación financiera en América Latina y el Caribe, Situación actual $y$ perspectivas, https://www.oecd.org/daf/fin/financial-education/OECD_CAF_Financial_ Education_Latin_AmericaES.pdf 